\title{
The Role of Dashboards in Long Lasting Insecticidal Nets (LLIN) Campaigns: A Pilot Report from Nigeria
}

\begin{abstract}
Akubue Augustine Uchenna ${ }^{1 *}$, Co-Authors: Jalal-Eddeen Abubakar Saleh ${ }^{1}$, Dr. Abdullahi Saddiq ${ }^{1}$, Dr. Ugwunweze Jacinta Ifeyinwa $^{2}$ Ukor Nkiruka Calista ${ }^{3}$, Dr. Ekweremadu Isaac ${ }^{4}$, Engr. Ikeonu Caroline Obianuju ${ }^{5}$, William Komakech ${ }^{6}$, Ada Erinne ${ }^{6}$ Angela Anwagom Izegbune ${ }^{6}$, Abonyi Isaiah Chimezie ${ }^{7}$, Dr. Abonyi, Dominic Ojiabor ${ }^{8}$, Joseph Akam Ingwu', Akpelu Ugochinyere Alvana ${ }^{10}$, Opara Emmanuel Chinedu ${ }^{10}$ John Anderson $^{11}$, Rex Mpazanje ${ }^{1}$, Alemu Wondimagegnehu ${ }^{12}$, Dr. Bala Mohammed Audu $^{13}$ Prof. Amadi Agwu Nkwa ${ }^{14}$
\end{abstract}

${ }^{1}$ Communicable and Non-Communicable Diseases Cluster World Health Organization, Abuja, Nigeria.

${ }^{2}$ Senior Register Public health departments, Federal Teaching Hospital, Abakiliki, Ebonyi state, Nigeria.

${ }^{3}$ Health System Strengthening Cluster, World Health Organization, Abuja, Nigeria.

${ }^{4}$ Catholic Relief Service, Abuja Nigeria.

${ }^{5}$ Zonal data management unit for Expanded Programme on Immunizations Cluster, WHO, Enugu.

${ }^{6}$ Expanded Programme on Immunizations Cluster, World Health Organization, SEZ Nigeria

${ }^{7}$ Environmental Health Department, Nnamdi Azikiwe University, Awka, Nigeria.

${ }^{8}$ Environmental Health Registration Council of Nigeria, Abuja.

${ }^{9}$ Public Health Department, Cross-River Ministry of Health, Calabar Nigeria.

${ }^{10} \mathrm{PhD}$ Student, Environmental Health Science, Uturu, Abia state.

${ }^{11}$ Postgraduate Department of Medical Education, Brighton \& Sussex Medical School, UK.

${ }^{12}$ WR, World Health Organization Country Office, Abuja, Nigeria.

${ }^{13}$ National Malaria Elimination programmes, Abuja, Nigeria.

${ }^{14}$ Environmental Health, Federal University of Technology, Owerri, Nigeria.

"Corresponding author: Akubue Augustine Uchenna, Non-Communicable and Communicable Diseases Cluster, World Health Organization, Abuja, Nigeria. Tel: +2348036591679; Email: akubueaugustine@yahoo.com

Citation: Uchenna AA, Saleh JA, Saddiq A, Ugwunweze J I, Ukor NC, et al. (2018) The Role of Dashboards in Long Lasting Insecticidal Nets (LLIN) Campaigns: A Pilot Report from Nigeria. J Community Med Public Health: CMPH-134. DOI: 10.29011/2577$2228 / 100034$

Received Date: 16 May, 2018; Accepted Date: 21 May, 2018; Published Date: 28 May, 2018 


\section{Abstract}

Background: Although there are various malaria intervention measures, the Long-Lasting Insecticidal Nets (LLIN) are considered as the most cost-effective intervention measure for malaria endemic countries. This study critically aims to look at the role of dashboards in the 2017 LLIN replacement campaigns that took place across five states (Adamawa, Edo, Imo, Kwara, and Ondo) in Nigeria.

Methods: This study conducted an in-depth review of the technical assistance provided by the WHO National Professional Officers on malaria to the five states that implemented the LLIN replacement campaign in 2017 in Nigeria - Adamawa, Edo, Imo, Kwara, and Ondo. Dashboards as strategic monitoring tools were deployed at stages to monitor the pre-implementation.

The findings from the dashboards at different stages of the campaign were analysed using the SPSS version 24.

Results: The result shows that out of 17 variables on the pre-implementation dashboards, the gaps flagged-off using the dashboard at 3 weeks' pre-implementation in the implementing states dropped from a maximum of 88.2\% (15/17) in Kwara state, $82.3 \%$ (14/17) in Edo state, 76.5\% (13/17) in Ondo state, and 70.6\% (12/17) in Adamawa and Imo states respectively to $0 \%(0 / 17)$ across all the states at three days to the campaign implementation. Also documented is the progressive increase in the proportion of distribution points (DPs) with LLINs by days of implementations from $32 \%$ to $45 \%, 68 \%, 80 \%$ and $100 \%$ in dayl, day2, day3, day 4 and day 5 respectively.

Conclusion: On the overall, this study further underscores the need to scale up the use of the LLIN dashboards that would be monitored using a 'virtual situation-room' with the technical input from the WHO NPOs on a real-time basis. This innovation in the malaria control programme in Nigeria would promptly flag-off key issues that potentially affect the quality of the LLIN campaign in Nigeria.

Keywords: Dashboards; Long-Lasting Insecticidal Nets; Malaria; National Malaria Elimination Programme; Nigeria; WHO

\section{Introduction}

Malaria remains one of the major public health problems as well as the main cause of mortality among young children in sub Saharan Africa and Nigeria in particular. It is endemic throughout Nigeria with about $97 \%$ of the population at risk and an estimated 300,000 deaths annually. Malaria is responsible for $60 \%$ of outpatient visits and $30 \%$ of hospitalizations in children under five years old and contributes to about $11 \%$ of maternal mortality in Nigeria [1]. Several studies revealed LLIN/ITNs to be one of the most effective malaria vector control methods. Studies in Africa have reported $17 \%-63 \%$ reductions in child mortality due to use of ITNs. Large scale use of LLINs is recommended by WHO as a major component of Integrated Vector Management (IVM) to prevent malaria [2].

Several countries recently showed rapid improvements in equitable LLINs ownership and usage following high quality, mass, free distribution campaigns [3-6].

Although there are various malaria intervention measures, the Long-Lasting Insecticidal Nets (LLIN) are considered as the most cost-effective intervention measure for malaria endemic countries [3]. Other available malaria preventive measures, as recommended by the World Health Organization (WHO) to achieve control and elimination, include: intermittent prophylactic treatment in pregnancy (IPTp), Intermittent Prophylactic Treatment in Infants (IPTi), Indoor Residual Spray (IRS), Seasonal Malaria Chemoprophylaxis (SMC), and, recently, the use of malaria vaccine.

The Distribution of LLINs through mass campaign is a key strategy for scale-up of ITN use, prescribed by the National Malaria Strategic Plan and Guidelines on Implementation of ITN in Nigeria [2]. Mass Campaigns are to be implemented in three phases; the first, the pre-implementation Phase, must involve "High Quality Guided Activities Which Requires Proper Monitoring Before Actual Distributions of Llins Can Guarantees Coverage and Access" [2].

Dashboard, in a broad sense, is an information management tool that visually tracks, analyses and displays key performance indicators (KPI), metrics and key data points to monitor the health of a business, department or specific process. They have wide application, including health programmes where they are customizable to meet the specific needs of a project or interventions. Behind the scenes, a dashboard interfaces with different agreed activities and outcomes, and, on the surface, displays the data in the form of tables, line charts, bar charts and gauges. A data dashboard is the most efficient way to track multiple data sources because it provides an easy-to-interpret, central location for monitoring and analysing performance. Real-time monitoring reduces the hours of analysing and long line of communication that previously challenged interventions [7]. A dashboard is a summary report - a snapshot picture - often based on status reports provided by projects, business change and "Business As Usual" (BAU), aimed 


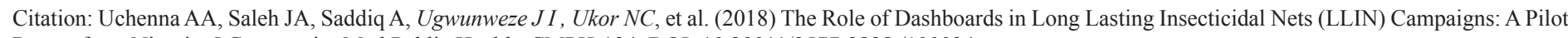
Report from Nigeria. J Community Med Public Health: CMPH-134. DOI: 10.29011/2577-2228 /100034

at showing where we need to focus attention and make decisions about issues. Dashboard is about reporting how well transition is going and how effectively outcomes are being achieved [8]. And can be seen as a business intelligence tool used to display data visualizations in a way that is immediately understood with aim of identifying and addressing gaps timely.

Dashboards are data visualization tools that allows all users to understand the analytics that matter to their business, department or project. Even for non-technical users, dashboards allow them to participate and understand the analytics process by compiling data and visualizing trends and occurrences. Data dashboards provide an objective view of performance metrics and serve as an effective foundation for further dialogue.

Types of KPIs and metrics tracked using data dashboards are based on the activities to monitor. Deciding which KPIs and metrics to track on a data dashboard is an important part of designing a dashboard. It's important to remember that dashboards must be built as a direct response to the needs of your team and organisation. The versatility of dashboards makes them useful for any organisation and department, including, but not limited to: executives, sales, marketing, social media, SaaS, development, supply, call centres and healthcare. Data dashboards track the KPIs and metrics unique to your area of business, and ultimately the ones that meet your specific analytic needs [8]. Dashboards often provide at-a-glance views of KPIs relevant to a particular objective or process on the other, "Dashboards" are another name for "progress report" or live "Report".

Dashboards can be strategic, analytical, operational, or informational [9]. Strategic dashboards support managers at any level in an organization, and provide the quick overview that decision makers need to monitor the health and opportunities of the business. Dashboards of this type focus on high level measures of performance, and forecasts. Strategic dashboards benefit from snapshots of data (daily, weekly, monthly, and quarterly) that are not constantly changing from one moment to the next.

In addition, dashboards allow for constant communication between all levels of a business. Data dashboards can be accessed by as many users as an organisation chooses, so that need to know information is at everyone's fingertips. No longer have do executives and managers need to request data from analysts. Analysts can work in the background to ensure a smooth operation, while users access this information whenever they want

Dashboard has wide applications for different organizations and programmes, and everyone uses data dashboards differently. Firstly, users need to know that dashboard definition is dependent on the role it plays within an organization. Not all dashboards serve the same purpose, which is why it is important that users understand what KPIs to track and why. The overall use depends on: what kinds of questions do dashboards answer? What types of data are tracked on dashboards and why? How dashboards interact and when to use the information from the dashboard[7]. Application of dashboards is designed to help manage and monitor programmes. Dashboards can have multiple applications, designed to cover the needs of different programmes in one organization. Whilst applications cover programme indicators, they are focused at the operational or activity level. This means that dashboards provide a tool to help both monitor (from the M\&E perspective) and manage (from the programme perspective) the programme from start to finish. Taking this holistic approach integrates indicators and data quality directly into the day to day activities of the team implementing the programme. It also provides project management tools that are customized for the programme itself [10].

In Nigeria, the first use of dashboard to monitor health programmes started with the National Primary Health Care Development Agency (NPHCDA) in collaboration with African Field Epidemiology Network-National Stop Transmission of Polio (AFENET-NSTOP) and other immunisation partners. The project "DHIS2 RI Dashboard Implementation Project" began in November 2014 with Kano as a pilot with the aim to track routine Immunization activities and outcomes [11]. The over-arching goals of this project are to improve the quality of RI data being generated and encourage the use of this data for action and decision-making, whilst promoting ownership at different levels. Specifically, the project aims to ensure key RI indicators, not previously available on DHIS2 national instance, are captured and made accessible on a DHIS2 RI dashboard [11].

A good dashboard looks at what data you want, where you can get it, gets agreement on what stakeholders want and establishes an agreed reporting schedule and targets. It must also understand who needs the information and work with them to agree on a common set of cleansed data. A dashboard is an efficient communicator as it ensures that messages get across at all levels. Good dashboards need to get your message or information across briefly, so a dashboard of any sort should have visuals, diagrams, tables and charts - rather than text. Visuals should convey the message in a self-explanatory way without the need for the audience to read lots of supporting narrative, focus on the quality of the metrics rather than quantity. It should provide an illustration of the "Delivery Health" of the project, programme or portfolio, e.g. how closely the actual schedule of output compares to the planned. A comparison of what is actually happening against what is planned, helps shift people from old business models and habits to practices referred to as" BAU business change", and is the main reason behind every good dashboard [7-10].

Its wide applications and documented successes made in immunization in Nigeria prompted its application in the LLINs 
distribution campaigns in Nigeria across the five states under study.

It is based on its wide applications and documented successes made in immunization in Nigeria that prompted and underscores its application in the LLINs distribution campaigns across the five states in Nigeria.

\section{Implementation Process of the Dashboard}

This study conducted an in-depth review of the use of dashboard as strategic tools by WHO National Professional Officers in providing technical assistance on malaria to the five states that implemented the LLIN replacement campaign in 2017Adamawa, Edo, Imo, Kwara, and Ondo (Figure 1). The campaign, rolled out in phases across the six states, took place between April and December 2017.

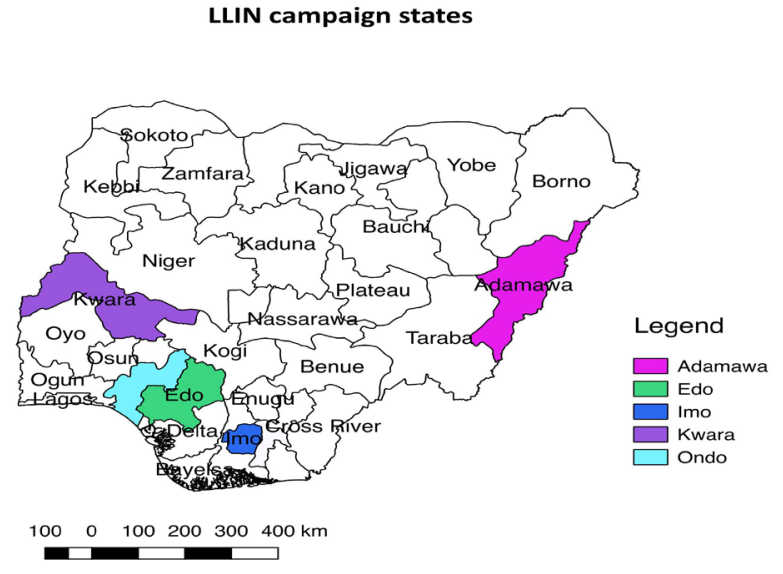

Figure 1: QGIS showing the five implementing states during the LLIN campaign.

WHO Nigeria, as part of its technical support to the National Malaria Elimination Programme (NMEP) for, the first time developed and applied periodically a dashboard to assess the preimplementation activities at 3 weeks, 1 week, and 3 days prior to the 2017 LLIN replacement campaign across the implementing states.

Development of the dashboard followed the findings from central desk reviews of different stages of all previously implemented LLINs campaign in Nigeria by All RBM partners. The various stages of LLINs distribution were stratified into 17 different activities as agreed by all RBM partners involved in campaigns in Nigeria. The seventeen variables considered in the dashboard are Effectiveness of state task force on implementation, effectiveness of LGA task force on implementation, adherence to implementation schedule of activities, planning meeting held, evaluation of micro planning, daily implementation work plan review, availability of plan for hard-to-reach areas, logistics availability, assessment of state level training of personnel, assessment of LGA level training of personnel, personnel availability at their duty post, quality and compliance to the developed supervisory work plan, efficiency of social mobilization committee (state and LGA), availability of IEC materials, Quality of the key messages of jingles and radio programmes, plans for flag-off at state and LGA levels, and availability of quality LLIN distribution work plan. All the RBM partners developed measuring and assessment indicators for each of these seventeen (17) pre-implementation activities. WHO then developed a dashboard to monitor the extent of activities implementations before LLINs distribution (Implementation phase) with the aim of tracking progresses and flagging up gaps capable of undermining the campaigns. These various activities and challenges identified and captured in dashboard were used for advocacy and needs assessment visits to the implementing states where the dashboards were to be implemented. This was followed by training of state, local government area (LGA) and health facility officers on the revised LLINs campaign activities and on how best to use the dashboards. The dashboard was piloted by WHO in two LGAs and issues raised were corrected before being deployed to the field periodically as data tools for the assessment of these activities were identified and agreed by all partners at 3 weeks, 1 week and 3 days intervals before the LLINs actual implementations.

After each assessment, the gaps and progress and challenges identified were shared among stakeholders' in meetings, the way forward were charted and specific actionable plans with timeframe were developed and feedbacks given to all levels on the results of the evaluation before the next assessment was done. By using this tool as guide, the progresses and challenges of pre-implementation activities' were monitored from one period or stage to other, until all the challenges were addressed three days before the actual implementations. The success of dashboard relies on the data quality generated by stakeholders, the quality of supportive supervision training, supportive supervision, and the clarity of KPI measured, policy drive, efficiency of periodic evaluation reports, and timely feedback and implementation of recommendations made after each review.

\section{WHO played the Following Vital Roles in the Implementation of Dashboard}

- Provided technical support toward the development of LLINs dashboard,

- Led the development of training modules for dashboard,

- Provided general coordination of implementation of dashboard,

- Provided technical support for training at state and national levels; 
- Coordinated provision of national feedback to states and follow-up with states to ensure recommendations are implemented at all levels,

- Coordinated and led advocacy and needs assessment visits to states prior to training,

- Coordinated presentation of periodic updates on the outcome to national, state, LGAs and IVM working groups at all levels.

- Most importantly, documented other activities not considered during the dashboard designs and shared experiences as lessons learnt for further campaigns.

- The findings from the dashboards at different stages of the campaign were analysed using the SPSS version 24.

\section{Results/Findings}

The findings from the dashboards at different stages of the campaign were analysed using SPSS version 24 .

Results: The result shows that out of 17 variables on the preimplementation dashboards, the gaps flagged-up using the dashboard in the implementing states dropped from a maximum of $88.2 \%(15 / 17)$ in Kwara state, $82.3 \%(14 / 17)$ in Edo state, $76.5 \%(13 / 17)$ in Ondo state, and $70.6 \%(12 / 17)$ in Adamawa and Imo states respectively at 3 weeks' pre-implementation, to $0 \%(0 / 17)$ across all the states at three days prior to the campaign implementation. (Table 1).

\begin{tabular}{|c|c|c|c|c|}
\hline \multirow{7}{*}{} & & $\begin{array}{c}3 \text { weeks } \\
\text { pre-implem } \\
\text { entation } \\
\text { dashboard }\end{array}$ & $\begin{array}{c}\text { 1 week pre- } \\
\text { implementation } \\
\text { dashboard }\end{array}$ & $\begin{array}{c}3 \text { days pre- } \\
\text { implem } \\
\text { entation } \\
\text { dashboard }\end{array}$ \\
\hline \multirow{5}{*}{ States } & Adamawa & 12 & 4 & 0 \\
\cline { 2 - 5 } & Edo & 14 & 4 & 0 \\
\cline { 2 - 5 } & Imo & 12 & 4 & 0 \\
\cline { 2 - 5 } & Kwara & 15 & 4 & 0 \\
\cline { 2 - 5 } & Ondo & 13 & 4 & 0 \\
\hline
\end{tabular}

Table 1: Gaps flagged-off up in pre-implementation dashboard indicators at 3 weeks, 1 week, and 3 days.

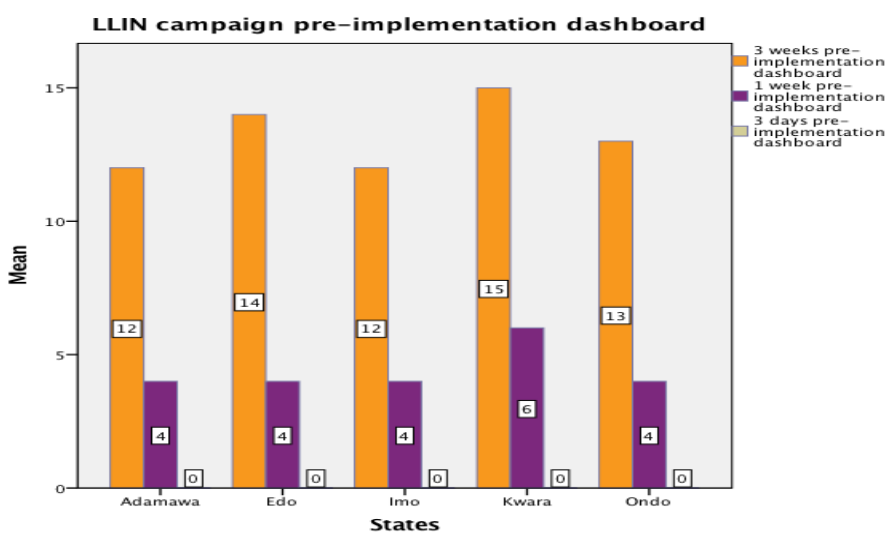

Figure 2: showing Bar chart of the gaps flagged-off up in the preimplementation dashboard indicators at 3 weeks, 1 week, and 3 days.

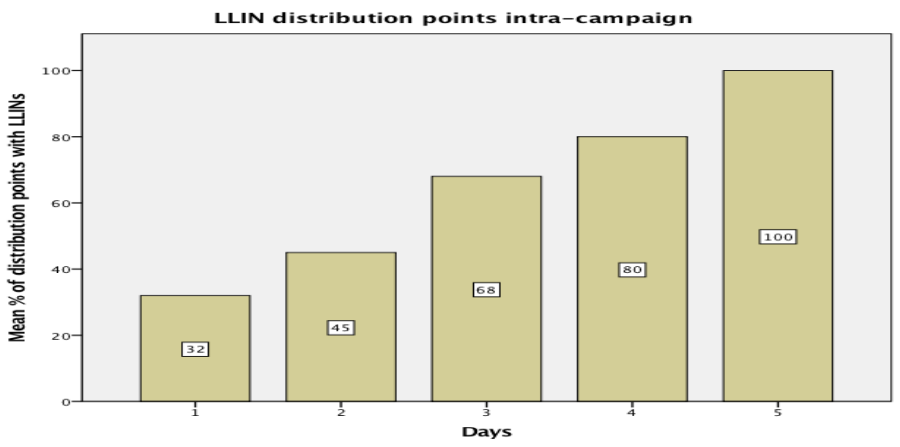

Figure 3: Showing the LLIN distribution points intra-campaign.

\begin{tabular}{|l|c|c|}
\hline \multicolumn{3}{|c|}{ Mean \% of distribution points with LLINs } \\
\hline \multirow{3}{*}{ Days } & 1 & 32 \\
\cline { 2 - 3 } & 2 & 45 \\
\cline { 2 - 3 } & 3 & 68 \\
\cline { 2 - 3 } & 4 & 80 \\
\cline { 2 - 3 } & 5 & 100 \\
\hline
\end{tabular}

Table 2: showing mean $\%$ distribution points with LLINs intracampaign. 


\section{Discussions}

This study aims to look critically at the role of dashboards in the 2017 LLIN replacement campaigns that took place across five states (Adamawa, Edo, Imo, Kwara, and Ondo) in Nigeria. The result shows that out of 17 variables on the pre-implementation dashboards, the gaps flagged-off across the implementing states have reduced from a maximum of $88 \%(15 / 17)$ in Kwara state at 3 weeks to $0 \%(0 / 17)$ in all the states at day three to the campaign implementation. This is in line with expectations of the dashboard application.

Our experiences of setting up this and working with partners at all levels and in all five locations taught us the following useful lessons:

- Establishing cordial and effective lines of communication between Dashboard personnel and local staff.

- The need to ensure accuracy and prompt recording and transmission of data from localities to Dashboard HQ.

- The need for high quality training of staff to input data and interpret Dashboard indicators.

- The value of giving (positive) feedback from DHQ to staff in the field.

This studies shows that dashboard aided in the successful monitoring of the 17 pre-implementation variables identified by stakeholders to be useful for successful campaign. Applying dashboard also demonstrated its importance in measuring progresses toward improving the LLINs campaign since it has shown to be very helpful as a comprehensive tool for measuring multiple dimensions of the system [19]. Development and implementation of the dashboard to assess LLINs campaign allowed national program managers to monitor the key net campaign indicators at all levels of implementations which was also noted in the [19]. Dashboard therefore should be considered as a very powerful tool for monitoring health interventions as demonstrated in this study and other studies done in immunization in Nigeria [19].

The result of this study also shows that out of 17 variables on the pre-implementation dashboards, the gaps flagged-off using the dashboard at 3 weeks pre-implementation in the implementing states dropped from a maximum of $88.2 \%(15 / 17)$ in Kwara state, $82.3 \%(14 / 17)$ in Edo state, $76.5 \%$ (13/17) in Ondo state, and 70.6\% $(12 / 17)$ in Adamawa and Imo states respectively to $0 \%(0 / 17)$ across all the states at three days to the campaign implementation.
Similar results of dashboard use as good monitoring and tracking tool in Nigeria have been demonstrated in immunization programme [20]. In Nigeria, for example, monitoring vaccine stock availability via dashboards increased the percentage of stores with adequate amounts of stock from 34 per cent to 81 per cent. Similarly, in four provinces of Mozambique, the generation of dashboards, alerts and reports led to a reduction in vaccine stock-outs from approximately 35 per cent to below 3 per cent and increased cold chain uptime from around 40 per cent to above 90 per cent. In India's Karnataka State, the introduction of simple supply chain dashboards and a feedback 'bulletin board' resulted in a better than 62 per cent reduction in the time needed to resolve vaccine stock-outs and minimum stock issues, contributing to a marked increase in vaccine availability [20]. Also noted in this study is the progressive increase in the proportion of distribution points with LLINs availability by days of implementations from $32 \%, 45 \%, 68 \%, 80 \%$ and $100 \%$ for day 1 , day 2 , day 3 , day 4 and day 5 respectively. This clearly shows that dashboard is useful in tracking team or personnel performances and commodity stock-outs during LLINs campaign. The study corroborated the evidence therein where it was used to track vaccination teams which provided significant feedback during polio campaigns that enabled supervisors to evaluate performance of vaccination teams [21]. Evaluating team performances is very important since most of low LLINs coverage during campaign is attributed majorly to team performance and commodity stock outs arising from gaps in logistic during campaigns.

This study demonstrated that dashboards allows effective tracking of performance in an engaging manner, and support timely interventions and can be easily used within cross-agency collaborations (e.g. collective impact initiatives) to track shared measures of change Unlike infographics where the information displayed is generally static, data dashboards are dynamic, with information being regularly refreshed and updated, often in 'real time' [23].

Application of dashboard with proven good result has been demonstrated even in clinical settings as it was used to determine the effect of the Maternal Newborn Dashboard on six key clinical performance indicators in the province of Ontario, Canada [24].

The study also shows lack of LLINs and other commodities at all distribution points until the last day of implementation which was attributed to the failure of logistic implementation plans that always result in stock-out of commodity at that levels. Although there may be robust logistic plans during pre-implementation of campaign, its implementation is always a problem sometimes due to lack of adequate transport system for micro-positioning of nets from state dry store to LGAs and DPs at ward levels which is in line with others studies done in Nigeria [28][29][30]. 


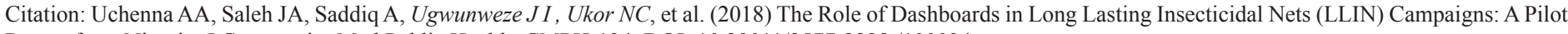
Report from Nigeria. J Community Med Public Health: CMPH-134. DOI: 10.29011/2577-2228 /100034

\section{Conclusion}

Our study taught us valuable lessons about setting up and using Dashboards in implementing malaria programmes. The results were very encouraging in demonstrating the effectiveness of Dashboards in monitoring LLINs campaign real -timewhile being used as prompt tool for addressing identified programme gaps. We also learnt the importance of establishing efficient and cordial relationships at all links from DHQ to local personnel involved in programming. On the overall, this study further underscores the need to scale up the use of the LLIN dashboards that would be monitored using a 'virtual situation-room' with the technical input from the WHO National Professional Officers on a real-time basis. Use of dashboard in LLINs promotes high quality Pre-implementation and implementation activities that resulted in high net retention and redemption rates recorded during the campaign across the states. This innovation in the malaria control programme in Nigeria would promptly flag-off key issues that potentially affect the quality of the LLIN campaign in Nigeria.

\section{Acknowledgements}

The Dashboard tool deployed by the World Health Organization during the LLIN campaign is part of the Technical Assistance given to the National Malaria Elimination Programme (NMEP) in Nigeria. We declare that there is no any potential conflict of interest or royalty associated with the research. We declare that there is no funding received from any source for this research than WHO funding for technical support.

\section{References;}

1. Uchenna AA, Chinedu OA, Anderson J, Saleh JE, Saddiq A, et al. (2018) Assessing the Knowledge, Practice and Attitudes (KPA) of Traditional Healers in Malaria Control Programme in Nsukka zone of Enugu State Nigeria: Journal of Health, Medicine and Nursing.

2. Steven F (2006) Information Dashboard Design: The Effective Visual Communication of Data.

3. Mark S, Yireh Ltd (2018) Essentials for a Project, Programme or Portfolio Dashboard: Dashboards, Governance, MoP, MSP, P3O.

4. Explore AXELOS Best Practice.

5. What is a data dashboard?

6. kwantu.net/components/2015/8/1/application

7. nphcda.gov.ng/dhis2-routine-immunisation-dashboard-implementation-project/Jun 10, 2017.

8. Hsien-Yeang S, Lyn MS (2014), developing a dashboard to help measure and achieve the triple aim: A population-based cohort study. BMC Health Serv Res 14: 363.
9. Berwick DM, James B, Coye MJ (2003) Connections Between Quality Measurement and Improvement: Med Care 41: I30-I38.

10. Martin L, Nelson E, Lloyd R, Nolan T (2007) Whole System Measures. Massachusetts: Institute for Healthcare Improvement $(\mathrm{IHH})$ Innovation Series White Paper.

11. Leape LL, Kabcenell Al, Gandhi TK, Carver P, Nolan TW, et al (2000) Reducing Adverse Drug Events: Lessons from a Breakthrough Series Collaborative. Jt Comm J Qual Improv 26: 321-331.

12. Wilson T, Berwick DM, Cleary PD (2003) What Do Collaborative Improvement Projects Do?: Experience from Seven Countries. Jt Comm J Qual Saf 29: 85-93.

13. Berwick DM, Nolan TW, Whittington J (2008) The Triple Aim: Care, Health, and Cost. Health Aff (Millwood) 27: 759-769.

14. Federal Ministry of Health [Nigeria] (2009) Guidelines for the implementation of ITNs in Nigeria. Abuja: NMCP.

15. Akubue AU, Ogboi S, Oketah AC, Jalal-Eddeen S, Saddiq A et al. (2013) Baseline Assessment of Distributed LLINs and Utilization for Malaria Control Programme in Enugu State SEZ, Nigeria: Indian Journals of Applied Research. Journals 3.

16. Bonner K, Mwita A, McElroy PD, Omari S, Mzava A, et al. (2011) Design, Implementation and Evaluation of a National Campaign to Distribute Nine Million Free LLINs to Children Under Five Years of Age in Tanzania. Malar J 10: 73. |

17. Hightower A, Kiptui R, Manya A, Wolkon A, Vanden Eng JL, et al. (2010) Bednet Ownership in Kenya: The Impact of 3.4 Million Free Bed Nets. Malar J 9: 183.

18. Kulkarni MA, Vanden EJ, Desrochers RE, Cotte AH, Goodson JL, et al. (2010) Contribution of Integrated Campaign Distribution of LongLasting Insecticidal Nets to Coverage of Target Groups and Total Populations in Malaria Endemic Areas in Madagascar. Am J Trop Med Hyg 82: 420-425.

19. Alain P, Maya MV, van den Ent, Stephen S, Alan RH, et al. (2017) Monitoring Results in Routine Immunization: Development of Routine Immunization Dashboard in Selected African Countries in the Context of the Polio Eradication Endgame Strategic Plan. The Journal of Infectious Diseases 216: S226-S236.

20. https://www.technet-21.org/iscstrengthening/index.php/en/data-formanagement-documents-and-downloads/guidance-on-dashboards.

21. Kebba T, Pascal M, Sisay GT, Peter N, Tesfaye BE, et al. (2016) Tracking Vaccination Teams During Polio Campaigns in Northern Nigeria by Use of Geographic Information System Technology 20132015. J Infect Dis 213: S67-S72.

22. Upfill-Brown AM, Voorman A, Chabot-Couture G, Shuaib F, Lyons HM (2016) Analysis of Vaccination Campaign Effectiveness and Population Immunity to Support and Sustain Polio Elimination in Nigeria. BMC Med 14: 60.

\section{Data Dashboard.}

24. Weiss D, Dunn SI, Sprague AE, Fell DB, Grimshaw JM, et al. (2018) Effect of a Population-Level Performance Dashboard Intervention on Maternal-Newborn Outcomes: An Interrupted Time Series Study. BMJ Quality \& Safety 27: 425-436. 
Citation: Uchenna AA, Saleh JA, Saddiq A, Ugwunweze J I, Ukor NC, et al. (2018) The Role of Dashboards in Long Lasting Insecticidal Nets (LLIN) Campaigns: A Pilot Report from Nigeria. J Community Med Public Health: CMPH-134. DOI: 10.29011/2577-2228 /100034

25. Saleh JE, Uchenna AA, Saddiq A, Wondimagegnehu A, Mpazanje R, et al. (2018) Lots Quality Assurance Survey (LQAS) As a Strategy to Achieving Quality LLIN Campaigns: the Nigerian Experience. Open Access Library Journal.

26. Saleh JE, Uchenna AA, Saddiq A, Wondimagegnehu A, Mpazanje $\mathrm{R}$, et al. (un published paper) Quality Assurance (QA) Tool in Public Health Campaigns: A Look At the 2017 LLIN Replacement Campaign in Nigeria.
27. www.sfhnigeria.org/wp-content/uploads/2017/.../SFH_supplychain_ web_FINAL.pdf

28. apps.who.int/medicinedocs/documents/s20211en/s20211en.pdf

29. Allianceformalariaprevention.com/.../NG-Sokoto-Logistics-ProcessEvaluation-25021. 